\title{
Photovoltage and Photocatalyzed Growth in Citrate-Stabilized Colloidal Silver Nanocrystals ${ }^{\dagger}$
}

\author{
Peter L. Redmond,* Xiaomu Wu, and Louis Brus \\ Chemistry Department, Columbia University, New York, New York 10027
}

Received: February 6, 2007; In Final Form: March 16, 2007

\begin{abstract}
The photocatalyzed reduction of aqueous silver ions, by citrate adsorbed on silver nanocrystals, is studied on Formvar/carbon TEM grids and in photoelectrochemical cells. The reaction is characterized by transmission electron microscopy (TEM) monitoring of individual particles throughout the growth process. The photoinitiated growth on the silver particles is uniform and is not dependent upon the laser polarization. The potential of silver particle working electrodes is shown to shift negatively under irradiation in solutions of citrate. Adding silver ions to this system quenches the charging of the particle working electrode through charge transfer to the silver ions. The reaction is hypothesized to result from photoelectron transfer from adsorbed citrate to the silver nanoparticle. Nanocrystal growth occurs when this "stored" charge reduces silver ions in solution. Comments are offered on the photo-reformulation of colloidal nanocrystals into prisms reported in the literature.
\end{abstract}

\section{Introduction}

Plasmon excited electronic states in metallic particles are fundamentally different from normal molecular or semiconductor quantum dot excited states. Metallic plasmons are formally represented as a coherent superposition of a very large number of low-energy electron-hole pair, rather than a single electron excitation of the same energy as the photon. This superposition state has a macroscopic oscillator strength which creates strong local electromagnetic SERS enhancement. The coherent plasmon dephases within femtoseconds, producing a hot electron gas. ${ }^{1-3}$ After electronic thermalization, energy relaxation occurs by exciting lattice phonons within the particle in a matter of picoseconds.

Osgood et al. first demonstrated molecular photochemistry in the plasmon-enhanced near-field. ${ }^{4}$ Gas-phase dimethylcadmiuim photodissociated just above a cadmium nanocrystal surface to produce free cadmium atoms which then deposited on the particle, leading to particle growth along the direction of the laser polarization. Adsorbed molecules can undergo electron-transfer photochemistry. The initial plasmon dephasing has been described as decay into a single "hot" electron excitation with the energy of the absorbed photon. ${ }^{5-11}$ The strong SERS seen for chemisorbed molecules is thought to result from femtosecond transient "hot" electron localization on the surface molecule. ${ }^{12}$ Electron-transfer photochemistry was reported in the early colloidal SERS literature; spectra were observed to change as they were being acquired. ${ }^{13,14}$ Furthermore, studies have shown that high-intensity visible laser irradiation can cause the formation of silver and gold nanoparticles both in solution ${ }^{15}$ and on surfaces. ${ }^{16,17}$

More recently, Maillard et al. reported low light intensity photocatalytic surface reduction of aqueous silver ions in the plasmon near-field, leading to particle growth. ${ }^{18}$ Colloidal silver particle seeds were irradiated in a growth solution of silver nitrate and sodium citrate. At room temperature, the competing

\footnotetext{
† Part of the special issue "Kenneth B. Eisenthal Festschrift".

* To whom correspondence should be addressed. E-mail: plr2001@ columbia.edu.
}

thermal reduction of $\mathrm{Ag}^{+}$by the citrate ion was quite slow. In other experiments, Jin et al. ${ }^{19}$ discovered that normal room light stimulates colloidal citrate-stabilized silver nanocrystals to reform into larger nanoprisms without added $\mathrm{Ag}^{+}$. Remarkably, smaller nanoprisms can re-form into larger nanoprisms under near-IR excitation. ${ }^{20,21}$ While the subject of much study, the mechanism of these specific photo-reformations, as well as that of photodriven surface electron-transfer processes in general, remains poorly understood. ${ }^{22-25}$

This paper explores the mechanism of the Maillard et al. plasmon photocatalyzed growth. They irradiated solutions of ca. $8 \mathrm{~nm}$ silver seeds in the presence of silver nitrate and sodium citrate and found that the particles grew to shapes defined by the laser wavelength. The initial step was attributed to $\mathrm{Ag}$ plasmon-induced photo-oxidation of adsorbed citrate, injecting an electron into the silver nanocrystal. Citrate itself does not absorb the light. This charge-transfer photo-oxidation is irreversible due to fast decarboxylation of the oxidized citrate. Theoretically, plasmon excitation focuses the incident optical field and the flux of undephased metallic electron-hole pairs, which form the plasmon, on the poles of the particles, as defined by the laser polarization direction. For this reason, it was hypothesized that the photoinduced reduction of silver ions would occur at the poles, leading to rod growth from round seeds. Actually, disc-shaped nanoprisms were observed. The authors reasoned that disc nanoprisms and not rods are observed because of rotational averaging of the growth direction in solution. This present paper tests the mechanism by directly observing the growth of stationary silver seeds deposited on dielectric surfaces. We find that the nonrotating particles grow uniformly and not as rods. To further explore the mechanism, we directly measure a change in the silver nanocrystal electrochemical potential due to photoinduced surface charge-transfer processes.

\section{Experimental Section}

Citrate-stabilized silver nanoparticles, as employed previously in hundreds of SERS papers, ${ }^{26}$ were synthesized using the LeeMeisel variation ${ }^{27}$ of the classic Turkevich procedure. Citrate 


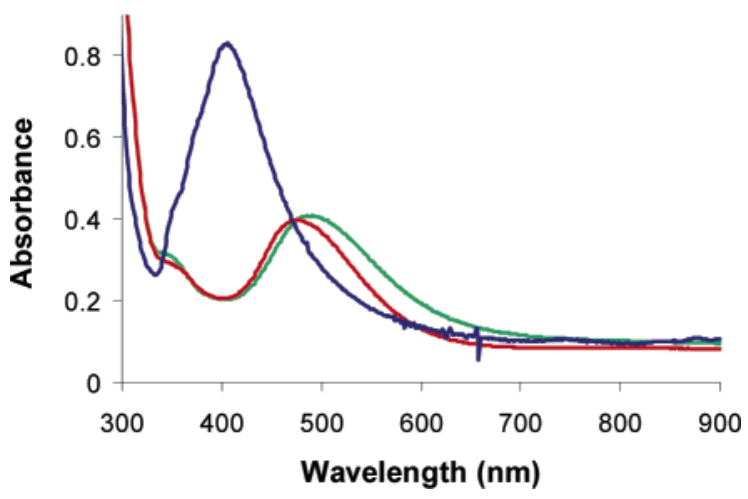

Figure 1. A UV-vis spectrum. The blue line is the colloidal silver nanoparticle solution, the green line is the silver particle electrode after annealing, and the red line is the silver particle electrode after repeated charging and discharging cycles in a solution of $500 \mu \mathrm{M}$ sodium citrate and $0.1 \mathrm{M}$ potassium nitrate (see Supporting Information).

acts as both a stabilizing and reducing agent. Briefly, a solution of $27 \mathrm{mg}$ of $\mathrm{AgNO}_{3}$ in $150 \mathrm{~mL}$ of Nanopure water was brought to a vigorous boil in a $250 \mathrm{~mL}$ round-bottom flask. Then, $3 \mathrm{~mL}$ of $2 \%$ sodium citrate by weight was quickly injected into the flask. The solution was allowed to reflux for $2 \mathrm{~h}$. Figure 1 shows a UV-vis spectrum of the silver nanoparticle solution. TEM examination of the particle solution showed that the particles were, on average, $25 \mathrm{~nm}$ in radius.

To monitor the photoinduced growth of the particles, they were deposited on Formvar/carbon TEM grids as follows: $20 \mu \mathrm{L}$ of the prepared solution was allowed to slowly evaporate on the grid, which was then dipped in Nanopure water to remove any remaining salt and allowed to dry again. The photoinduced growth was carried out by placing a particle-coated TEM grid in a solution composed of $100 \mu \mathrm{L}$ of Nanopure water, $50 \mu \mathrm{L}$ of $10 \mathrm{mM}$ sodium citrate, and $50 \mu \mathrm{L}$ of $5 \mathrm{mM} \mathrm{AgNO}_{3}$. The grid was illuminated with Ar-ion laser light at 514 or $488 \mathrm{~nm}$ at normal incidence. TEM images of the same individual particles were obtained both before and after irradiation in solution.

Photoelectrochemical experiments were performed in a two compartment cell to ensure that the reference and counter electrodes were isolated from laser irradiation. A platinum foil was the counter electrode, and a gold wire served as a pseudoreference electrode. A $3 \mathrm{~cm}^{2}$ area working silver nanoparticle electrode was made by thermally evaporating $\sim 2.5 \mathrm{~nm}$ of silver onto an indium tin oxide (ITO)-coated glass slide (Thin Film Devices, film thickness $=140 \mathrm{~nm}$ ). The electrode was annealed in a convection oven in air at $\sim 120{ }^{\circ} \mathrm{C}$ to form well shaped nanocrystals from the evaporated silver. Figure S1 shows a scanning electron micrograph (SEM) and Figure 1 shows a UV/vis spectrum of a typical particle electrode. All photovoltage solutions were bubbled with $\mathrm{N}_{2}$ for 20 min to remove oxygen.

\section{Results}

Citrate-stabilized Ag nanocrystals deposited on nonconducting Formvar/carbon TEM films were immersed in a growth solution of $2.5 \times 10^{-4} \mathrm{M}$ silver nitrate and $5 \times 10^{-4} \mathrm{M}$ sodium citrate. At room temperature, the thermal reduction of $\mathrm{Ag}^{+}$by the citrate ion is quite slow; without irradiation, no growth was detected after $1 \mathrm{~h}$ by TEM comparison of the same individual particles before and after immersion. However, Figures 2 and 3 show the growth of individual silver particles on a TEM grid with very-low-intensity (ca. $0.1 \mathrm{~W} / \mathrm{cm}^{2}$ ) 488 or $514 \mathrm{~nm}$ illumination for a few minutes. These wavelengths are resonant with the plasmon absorption of these approximately round ca. $25 \mathrm{~nm}$ radius particles. Essentially, all particles of this size are observed

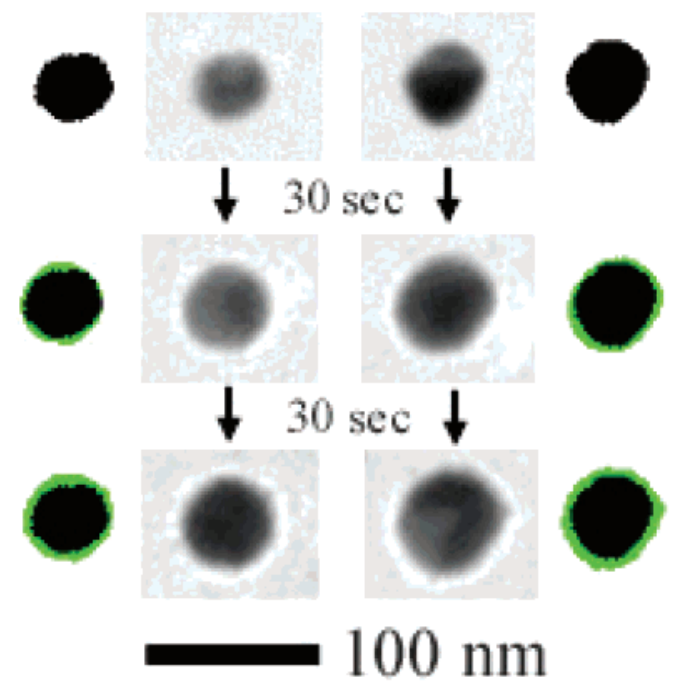

Figure 2. TEM images of silver particles before and after growth by $100 \mathrm{~mW} / \mathrm{cm}^{2}$ of $488 \mathrm{~nm}$ laser irradiation. The first row is with no irradiation. The second and third rows are for 30 and $60 \mathrm{~s}$ of irradiation, respectively. The first and fourth columns are outlines of the particles with black as the unreacted particle and green as the regions of growth.

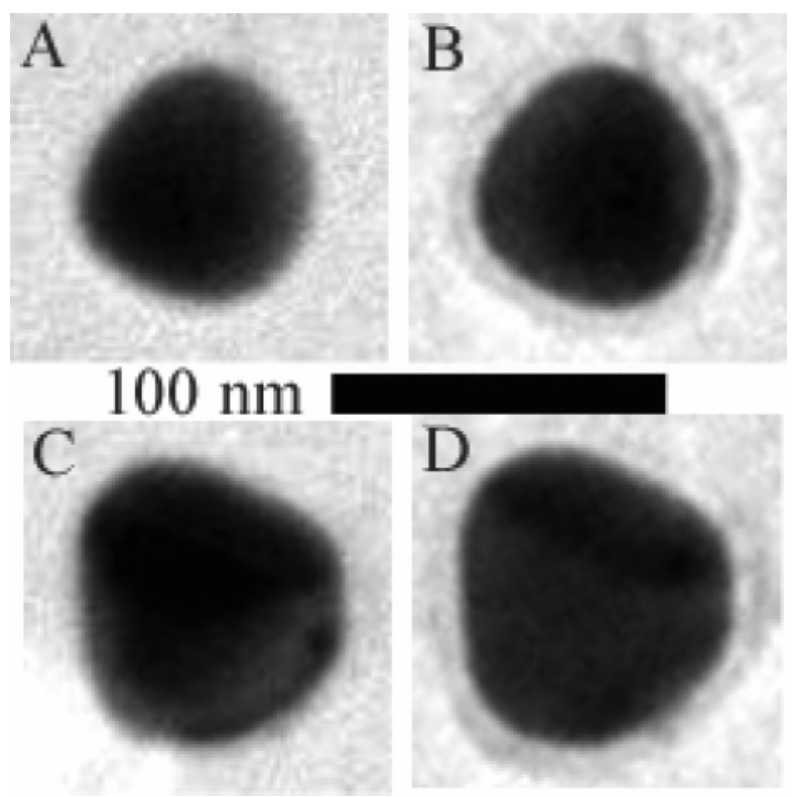

Figure 3. TEM images of a silver particle before and after growth by $100 \mathrm{~mW} / \mathrm{cm}^{2}$ of $514 \mathrm{~nm}$ laser irradiation for $5 \mathrm{~min}$. Particles A and C are before and particles $\mathrm{C}$ and $\mathrm{D}$ are after.

to grow. The growth is uniform within the plane of the adsorbed particle and independent of the laser polarization direction at both 514 and $488 \mathrm{~nm}$ excitation. Figures S2, S3, and S4 show the photoinduced growth of more particles. In 1 min at $488 \mathrm{~nm}$ laser wavelength excitation, the nanocrystal radius typically increased from 20 to $28 \mathrm{~nm}$. For irradiation of $10 \mathrm{~min}$ or more, the particles begin to touch each other. No growth under illumination occurs in the presence of citrate if $\mathrm{Ag}^{+}$is absent from solution.

In the mechanism outlined by Maillard et al., the first step is irreversible photo-oxidation of adsorbed citrate, presumably at the poles of the particle along the direction of the laser polarization, transferring an extra electron to the silver particle. If this were followed by immediate reduction of $\mathrm{Ag}^{+}$at the same spot, then the particle would grow only in this direction. However, we see growth in all directions. This suggests that the electron in the silver particle is long-lived and can reduce 


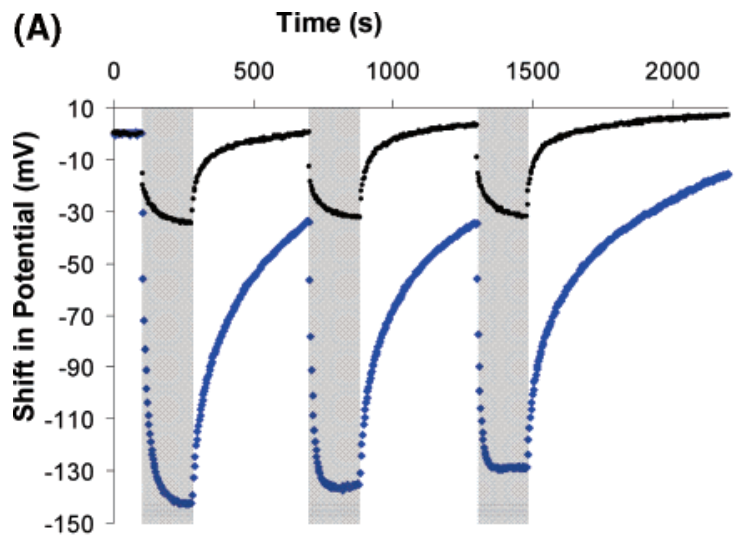

(B)

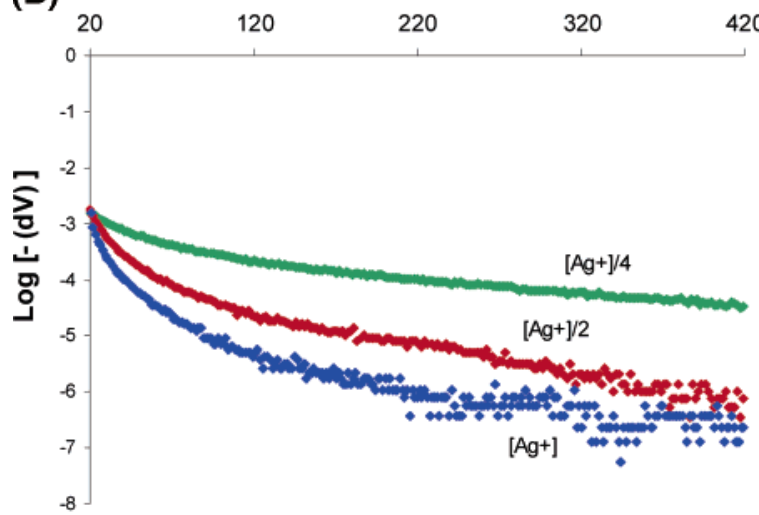

Figure 4. (A) Shift from rest potential of a silver particle working electrode in a solution of $500 \mu \mathrm{M}$ sodium citrate and $0.1 \mathrm{M}$ potassium nitrate. The black circles have $250 \mu \mathrm{M}$ silver nitrate in solution. The blue diamonds have no silver nitrate in solution. The shaded areas of the graph show when the laser is on. The unshaded parts show when the laser is off. The laser power was $100 \mathrm{~mW} / \mathrm{cm}^{2}$ at $488 \mathrm{~nm}$. (B) A semilog plot of the shift from rest potential versus time of nanoparticle electrodes after the laser has been turned off in solutions with different concentrations of silver ions. The blue line has 250, red has 125 , and green has $62.5 \mu \mathrm{M}$ silver nitrate. Each solution has $500 \mu \mathrm{M}$ sodium citrate and $0.1 \mathrm{M}$ potassium nitrate in addition to the silver ion concentrations.

adsorbed $\mathrm{Ag}^{+}$anywhere on the surface. Under irradiation, the steady-state Ag electrochemical potential would then shift negatively as electrons are accumulated, depending upon the slow, presumably thermal, rate of subsequent $\mathrm{Ag}^{+}$reduction on the surface. Thus, we attempted to directly detect a possible change in the Ag particle electrochemical potential using photoelectrochemical methods similar to those used by other groups for electrodes functionalized with deposited metal particles and carbon nanotubes. ${ }^{28-31}$

Thermally evaporated Ag particles of radius ca. $25 \mathrm{~nm}$ on ITO electrodes were irradiated in solution at the same light intensity as that in the growth studies above. In previous electrochemical Ostwald ripening experiments, we had shown that such adsorbed Ag particles equilibrate electrically with the conducting ITO substrate through a tunneling junction. Figure 4A shows the cell potential at zero current as a function of time in solutions of citrate, both with and without silver nitrate. Under irradiation, the Ag particle electrode steady-state potential shifts $-140 \mathrm{mV}$ in the citrate solution and $-35 \mathrm{mV}$ in the citrate and silver nitrate solution. Figure S5 shows the reproducibility of the voltage under many cycles. Following irradiation, the $\mathrm{Ag}$ electrode potential decays faster in the presence of increasing $\mathrm{Ag}^{+}$, as is shown in Figure 4B. No photoresponse was detected in electrolyte solutions of $0.1 \mathrm{M}$

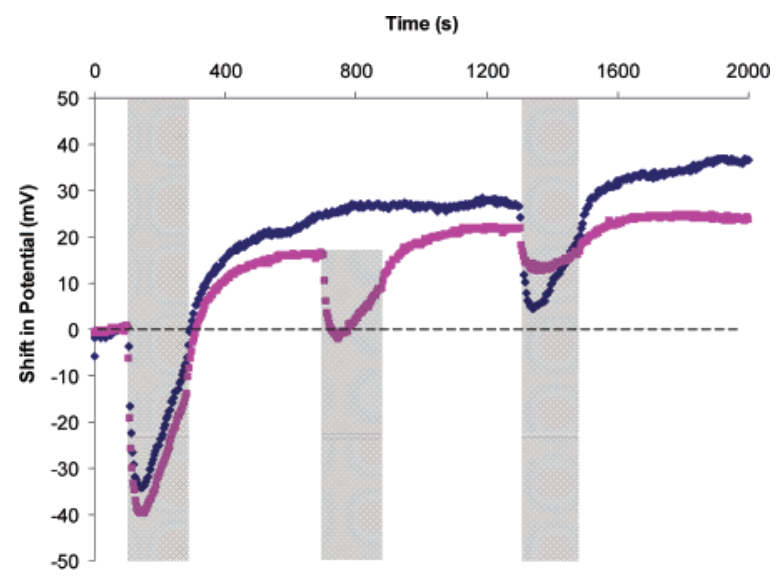

Figure 5. Shift from rest potential of a citrate-coated silver particle working electrode in a solution of $0.1 \mathrm{M}$ potassium nitrate. The shaded areas of the graph show when the laser is on. The unshaded parts show when the laser is off. The laser power was $100 \mathrm{~mW} / \mathrm{cm}^{2}$ at $488 \mathrm{~nm}$. Note: in the second laser-on cycle, the pink line is irradiated, and the blue diamonds are not. The pink squares are irradiated every $10 \mathrm{~min}$ for $3 \mathrm{~min}$, and the blue diamonds are irradiated every $20 \mathrm{~min}$ for 3 min.

potassium nitrate at our laser power densities, demonstrating that the process requires citrate. No photoresponse was observed for citrate solution in contact with an annealed ITO electrode without Ag particles. We conclude that the response is due to excitation of the Ag particle in the presence of citrate.

In the Maillard mechanism, adsorbed citrate is photo-oxidized. Presumably the adsorbed layer is in equilibrium with aqueous citrate. To investigate this, we prepared a sample in which the solution contained no free citrate. A silver nanocrystal electrode was first immersed in a $500 \mu \mathrm{M}$ solution of sodium citrate for $10 \mathrm{~min}$ and then rinsed with pure water and dried. The photoresponse in Figure 5 was measured in a $0.1 \mathrm{M}$ potassium nitrate solution, without aqueous citrate or $\mathrm{Ag}^{+}$. The response is very different. Experiments on two different electrodes are shown in pink and blue. In the pink trace, photovoltage is generated at the beginning of the first irradiation period. However, it decays under irradiation and does not reach a photostationary state as in Figure 4A. In the second and third irradiation period, the photovoltage is smaller, and the dark rest potential shifts positively. We assign the progressively weaker photovoltage to loss of surface citrate, without replacement by aqueous citrate. The rest potential shifts positively as the initially strong double-layer stabilization of negatively charged citrate is lost.

In this experiment, is the adsorbed citrate lost due to photooxidation or just due to thermal equilibration with the solution? In the blue trace, the experiment was repeated without the second irradiation. During the third irradiation period on the pink trace, the equivalent photovoltage on the blue trace was stronger. If the loss of photoresponse was due to thermal diffusion of surface citrate molecules into solution, with no photoeffect, then these peaks would have matched. This result suggests that it is the photo-oxidation of the adsorbed citrate layer that causes loss of the photoresponse.

\section{Discussion}

A. Irreversible Citrate Photo-Oxidation. Citrate undergoes both thermal and photochemical oxidation on silver through the following reaction ${ }^{32,33}$

$$
\text { citrate } \rightarrow \text { acetone-1,3-dicarboxylate }+\mathrm{CO}_{2}+2 \mathrm{e}^{-}
$$



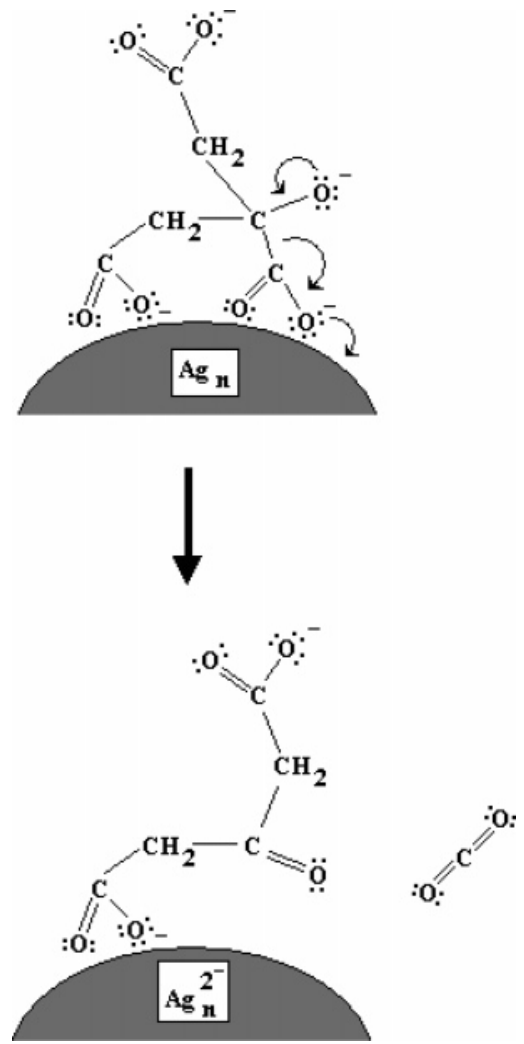

Figure 6. Proposed mechanism for the photo-oxidation of citrate through the donation of electrons into the silver nanoparticle and the creation of carbon dioxide.

Munro et al. proposed that citrate binds to silver particles, as is shown in the first step in Figure $6 .{ }^{34}$ Citrate sits on the silver nanoparticle with two of its' three carboxylic groups associating with the silver particle. The nucleophilic adsorption of the carboxylic anions causes fractional electron transfer into the silver particle. The third carboxylic anion is presented to the solution. Munro et al. suggested that the carboxylate that is directly attached to the central $\mathrm{sp}^{3}$ carbon oxidizes to carbon dioxide and acetonedicarboxylic acid. Beltramo et al. studied the related electrochemical oxidation of formic acid on a gold electrode and found that two electrons transfer from the adsorbed formate ion to the gold electrode, yielding carbon dioxide and a hydrogen ion. ${ }^{35}$ We hypothesize that the photo-oxidation of citrate proceeds through the mechanism shown in Figure 6. Excitation of the silver particle causes the "short-armed" carboxylic group of the citrate to donate two electrons into the silver. This causes the central carbon to transfer electrons to the carboxylate group, forming carbon dioxide. The oxygen group on the central carbon donates its free electrons to a double bond, forming an oxy group. The result is that two electrons are irreversibly donated into the particle, carbon dioxide is released, and acetonedicarboxylate remains on the particle. Munro et al. noted that acetonedicarboxylate is unstable and spontaneously decarboxylates to form acetoacetic acid. This mechanism, although speculative, illustrates how citrate can irreversibly react with the short-lived electronic excitation of the silver particle through the ejection of carbon dioxide.

B. Photovoltage. We attribute the photovoltage to irreversible electron transfer into the $\mathrm{Ag}$ particle due to photo-oxidation of adsorbed citrate. In essence, photo-oxidation causes the fixed surface oxide anion charge to become a mobile metallic electron, which then can equilibrate with the electrode. This is a photodriven "hot" hole reaction (most likely caused by decay of the plasmon excitation); at this low power density, particle laser heating is negligible. ${ }^{36,37}$ Note that we excite the broad plasmon feature with a single-frequency $\mathrm{CW}$ laser, which does not have the spectral width necessary to excite the complete, zero-order plasmon. Rather, we likely excite the coherent decay product of the plasmon, hot electron-hole pair(s).

In related experiments, Otto et al. systematically explored the electrochemical reactions of "hot" ballistic electrons injected electrically into thin Ag electrodes; such "hot" electrons were able to overcome over-potentials to induce electrochemical surface reactions which were inaccessible to "cold" electrons. ${ }^{38}$ Hot electron photochemistry of adsorbed molecules, following metal excitation, is also well-known in high-vacuum experiments. ${ }^{39}$

In our experiment, during irradiation, the potential becomes more negative and reaches a steady state where the rate of electron charging is equal to the rate of electron discharge, presumably through hydrogen reduction. When there is silver nitrate in the solution, the photoresponse of the system is lower because the particles can discharge their stored electrons to silver ions in solution. A quantitative analysis of the observed kinetics on ITO must await further systematic experiments and modeling. For a single colloidal particle, the negative photovoltage is an increase in the (photostationary) Ag surface double-layer potential. In the absence of free citrate in solution, adsorbed citrate is eventually consumed, and the initially strong, negative double-layer potential decreases.

In 1980, Henglein and Lilie showed that colloidal, sodium polyvinyl sulfate (PVS)-stabilized Ag nanoparticles can store negative charge created by surface radical reactions. ${ }^{40}$ They found that a $7 \mathrm{~nm}$ silver particle could hold up to 450 extra electrons. The discharge of stored electrons occurred through hydrogen reduction in acidic solution over a period of several minutes. The silver nanocrystal acts as a nanoelectrode. In our work, the stored electrons reduce silver ions in solution. The photovoltage and related capacitance are different in solution than on the ITO surface and depend upon particle shape. Nevertheless, following Henglein and Lilie, we can roughly estimate the stored charge using the simple capacitance formula for an isolated round metal particle

$$
\Delta V=\frac{Q}{4 \pi \epsilon R}
$$

Here, $V$ is the potential (with respect to the bulk solution) of a particle with charge $Q$ and radius $R$ in a dielectric medium of permittivity $\epsilon$. The equilibrium potential shift of the particle electrode in citrate solution without $\mathrm{Ag}^{+}$(the first peak in Figure $4 \mathrm{~A}$ ) is about $-140 \mathrm{mV}$. Using eq 2 , this corresponds to about 195 electrons stored in a $25 \mathrm{~nm}$ radius particle. There is one extra electron for every 20000 silver atoms in the particle.

In the single-particle growth experiments on dielectric Formvar substrates, the observed rate under irradiation corresponds to a radius increase from 20 to $28 \mathrm{~nm}$ in $60 \mathrm{~s}$. This corresponds to a reaction rate of about $182 \mu \mathrm{A} / \mathrm{cm}^{2}$ on each particle surface. A simple estimate shows this rate is not limited by $\mathrm{Ag}^{+}$ diffusion. ${ }^{41}$ This rate corresponds to 28500 photo-oxidations of citrate per particle per second (assuming two donated electrons per citrate). We estimate the total number of citrate molecules on a $20 \mathrm{~nm}$ particle to be 20000 (assuming the surface area required for each citrate molecule ${ }^{42}$ is $0.25 \mathrm{~nm}^{2}$ ). Using Mie theory, we calculate the absorption cross section of a $25 \mathrm{~nm}$ radius $\mathrm{Ag}$ particle in water to be $3.2 \times 10^{-16} \mathrm{~m}^{2}$ and the scattering cross section to be $1.2 \times 10^{-15} \mathrm{~m}^{2}$ at $488 \mathrm{~nm}$. At $0.1 \mathrm{~W} / \mathrm{cm}^{2}$, the estimated citrate photo-oxidation quantum yield 
is $\sim 0.5 \%$ based upon the combined Ag particle scattering and absorption cross sections. ${ }^{43}$

It is possible to estimate the potential shift required to account for the growth of the particles on Formvar using the ButlerVolmer equation. Using a cyclic voltammogram, we can relate the potential shift of a particle with the surface reaction rate (i.e., deposition rate). Figure S7 shows the Tafel current-voltage plot of a rough evaporated silver/ITO electrode (without annealing to form particles). From this, we derive the exchange current density and transmission coefficient of the Butler-Volmer equation; these parameters allow us to quantitatively convert growth rate into voltage shift. The experimental reduction rate corresponds to a potential shift of about $-130 \mathrm{mV}$. This estimate for Ag particles on Formvar/carbon is roughly consistent with the directly measured photovoltages on ITO in Figure 4A. Note that, in the Butler-Volmer equation, the reduction rate is exponentially dependent upon voltage; a negative shift of $140 \mathrm{mV}$ corresponds to an increase in the rate by a factor of 24 .

C. SERS, Photocoagulation, and Prism Growth. The standard silver particle preparation for surface-enhanced Raman scattering (SERS) is the citrate reduction of silver nitrate introduced by Lee and Meisel. ${ }^{27}$ Figure 4A shows that power densities as low as $0.1 \mathrm{~W} / \mathrm{cm}^{2}$ can significantly shift the electrochemical potential of such particles. In SERS studies, it is common to use power densities 100-1000 times higher. On rough Ag electrodes, the SERS intensity is known to vary with applied potential. ${ }^{44}$ It is possible that the photo-oxidation of citrate causes a particle photovoltage shift which, in turn, causes nonlinearities in the SERS intensity with respect to laser power. Additionally, this potential shift might explain some of the electron-transfer reactions observed to occur as SERS spectra are obtained.

Despite some ambiguity in the Jin et al. initial report in reference 20, Sun and Xia established that citrate stabilization is required in the room-light-induced nanoparticle-to-prism reformulation process. ${ }^{24}$ Bastys et al. noted the presence of an isosbestic point in the time-resolved UV/vis absorption spectra of the photoconversion, thus ruling out an Ostwald ripening mechanism. ${ }^{45}$ At room temperature and in the dark, the particle solutions are stabile because of the capping action of citrate. When formed in the absence of citrate, such borohydridereduced particles quickly aggregate. ${ }^{46} \mathrm{We}$ conjecture that room light cumulatively destroys the strong citrate double-layer kinetic stabilization of the initial colloid. In solution, many particles then coagulate and come into electrical equilibrium with each other. Particles in electrical equilibrium, in the absence of added $\mathrm{Ag}^{+}$, can re-form into one large structure due to the size dependence of the redox potential. ${ }^{47}$ Similar ideas could be used to describe the thermal oxidation of citrate in the thermal synthesis of silver nanoprisms. ${ }^{22,48}$ Finally, we note that a related irreversible photo-oxidation mechanism has been described for Ag filament formation following ultraviolet irradiation of formic acid-stabilized colloidal Ag particles by A. Rogach et al. in ref 33 .

\section{Conclusion}

These experiments help to outline the mechanism of citraterelated Ag nanocrystal photoreduction and prism photoreformulation experiments. Adsorbed citrate is irreversibly photo-oxidized by "hot" holes during plasmon excitation. Electrons accumulate on the Ag particle; the resulting negative potential shift can be directly measured for adsorbed particles in a photoelectrochemical experiment. Nearby $\mathrm{Ag}^{+}$ions in solution, if present, are thermally reduced by the shifted potential on a time scale of seconds, leading to particle growth. There is no relationship between the laser polarization direction and the direction of particle growth. In a citrate-stabilized Ag colloid (without added $\mathrm{Ag}^{+}$), we conjecture that low-intensity irradiation leads to cumulative citrate destruction and thus loss of doublelayer stabilization. The colloid photocoagulates, producing growth of one larger structure from many smaller nanoparticles in electrical equilibrium. Careful quantitative study is yet required to firmly establish these processes.

Acknowledgment. Lou Brus gratefully thanks Ken Eisenthal for numerous stimulating discussions of molecular and nanocrystal chemical physics over the years. We thank Andreas Otto for valuable comments on an earlier draft of this manuscript. This work has been primarily supported by the DOE Basic Energy Sciences FG02-98ER14861. We have used characterization facilities supported by the Columbia NSF Nanocenter CHE-010110655, by the New York Office of Science, Technology and Academic Research NYSTAR, and by the Columbia NSF MRSEC materials center DMR 02123547.

Supporting Information Available: SEM of the particle electrode, UV-vis of the particle electrode, more TEMs of particles before and after growth, a plot of the repeated charging and discharging of a particle electrode, a plot of the rate of discharge versus silver ion concentration, an alternative mechanism for the oxidation of citrate on silver, a Butler-Volmer plot and Tafel plot of a silver electrode. This material is available free of charge via the Internet at http://pubs.acs.org.

\section{References and Notes}

(1) Liau, Y.-H.; Unterreiner, A. N.; Chang, Q.; Scherer, N. F. J. Phys. Chem. B 2001, 105, 2135.

(2) Hu, M.; Hartland, G. V. J. Phys. Chem. B 2002, 106, 7029.

(3) Hodak, J. H.; Martini, I.; Hartland, G. V. J. Phys. Chem. B 1998 $102,6958$.

(4) Chen, C. J.; Osgood, R. M. Phys. Rev. Lett. 1983, 50, 1705

(5) Zhdanov, V. P.; Hagglund, C.; Kasemo, B. Surf. Sci. Lett. 2005, 599, L72.

(6) Douketis, C.; Haslett, T. L.; Shalaev, V. M.; Wang, Z.; Moskovits, M. Physica A 1994, 207, 352.

(7) Nolle, E. L.; Shchelev M. Ya. Tech. Phys. Lett. 2004, 30, 304.

(8) Lemann, J.; Merschdorf, M.; Pfeiffer, W.; Thon, A.; Voll, S.; Gerber, G. J. Chem. Phys. 2000, 112, 5428.

(9) Kennerknecht, C.; Hovel, H.; Merschdorf, M.; Voll, S.; Pfeiffer, W. Appl. Phys. B 2001, 73, 425.

(10) Merschdorf, M.; Pfeiffer, W.; Thon, A.; Voll, S.; Gerber, G. Appl Phys. A 2000, 71, 547.

(11) Link, S.; El-Sayed, M. A. J. Phys. Chem. B 1999, 103, 8410

(12) Otto, A.; Fukamata, M. Top. Appl. Phys. 2006, 103, 147.

(13) Jiang, J.; Bosnick, K.; Maillard, M.; Brus, L. J. Phys. Chem. B 2003, 107, 9964 .

(14) Andersen, P. C.; Jacobson, M. L.; Rowlen, K. L. J. Phys. Chem. B 2004, 108, 2148.

(15) Kurihara, K.; Kizling, J.; Stenius, P.; Fendler, J. H. J. Am. Chem. Soc. 1983, 105, 2574

(16) Monti, O. L. A.; Fourkas, J. T.; Nesbitt, D. J. J. Phys. Chem. B 2004, 108, 1604.

(17) Bjerneld, E. J.; Svedberg, F.; Kall, M. Nano Lett. 2003, 3, 593.

(18) Maillard, M.; Huang, P.; Brus, L. Nano Lett. 2003, 3, 1611.

(19) Jin, R.; Cao, Y. W.; Mirkin, C. A.; Kelly, K. L.; Schatz, G. C.; Zheng, J. G. Science 2001, 294, 1901.

(20) Jin, R.; Cao, Y. C.; Hao, E.; Metraux, G. S.; Schatz, G. C.; Mirkin, C. A. Nature 2003, 425, 487.

(21) Callegari, A.; Tonti, D.; Chergui, M. Nano Lett. 2003, 3, 1565.

(22) Sun, Y.; Mayers, B.; Xia, Y. Nano Lett. 2003, 3, 675.

(23) Tian, X.; Chen, K.; Cao, G. Mater. Lett. 2006, 60, 828

(24) Sun, Y.; Xia, Y. Adv. Mater. 2003, 15, 695

(25) Jia, H.; Zeng, J.; Song, W.; An, J.; Zhao, B. Thin Solid Films 2006 496, 281.

(26) Michaels, A. M.; Nirmal, M.; Brus, L. E. J. Am. Chem. Soc. 1999, 121,9932

(27) Lee, P. C.; Meisel, D. J. Phys. Chem. 1982, 86, 3391. 
(28) Barazzouk, S.; Hotchandani, S.; Vinodgopal, K.; Kamat, P. V. J. Phys. Chem. B 2004, 108, 17015.

(29) Hasobe, T.; Imahori, H.; Kamat, P. V.; Ahn, T. K.; Kim, S. K.; Kim, D.; Fujimoto, A.; Hirakawa, T.; Fukuzumi, S. J. Am. Chem. Soc. 2005, 127,1216

(30) Lahav, M.; Gabriel, T.; Shipway, A. N.; Willner, I. J. Am. Chem. Soc. 1999, 121, 258.

(31) Chapman, P.; Mulvaney, P. Chem. Phys. Lett. 2001, 349, 358.

(32) Sato, T.; Kuroda Shigeru, K.; Takami, A.; Yonezawa, Y.; Hada, H. Appl. Organomet. Chem. 1991, 5, 261.

(33) Rogach, A. L.; Shevchenko, G. P.; Afanas'eva, Z. M.; Sviridov, V. V. J. Phys. Chem. B 1997, 101, 8129.

(34) Munro, C. H.; Smith, W. E.; Garner, M.; Clarkson, J.; White, P. C. Langmuir 1995, 11, 3712.

(35) Beltramo, G. L.; Shubina, T. E.; Koper, M. T. M. ChemPhysChem 2005, 6, 2597.

(36) Redmond, P. L.; Walter, E. C.; Brus, L. E. J. Phys. Chem. B. 2006, $110,25158$.

(37) Lowe, L. B.; Brewer, S. H.; Kramer, S.; Fuierer, R. R.; Qian, G.; Agbasi-Porter, C. O.; Moses, S.; Franzen, S.; Feldheim, D. L. J. Am. Chem. Soc. 2003, 125, 14258.

(38) Diesing, D.; Kritzler, G.; Otto, A. Top. Appl. Phys. 2003, 85, 365.
(39) Vondrak, T.; Burke, D.; Meech, S. Chem. Phys. Lett. 2001, 347,

(40) Henglein, A.; Lilie, J. J. Am. Chem. Soc. 1981, 103, 1059.

(41) Estimated from equation 3 of: Lui, H.; Penner, R. M. J. Phys. Chem. B 2000, 104, 9131.

(42) Dagastine, R. R.; Grieser, F. Langmuir 2004, 20, 6742

(43) In molecules, the absorption cross section is orders of magnitude larger than the scattering cross section. Quantum yields from thermally equilibrated excited states are based upon the absorption cross section. In $25 \mathrm{~nm} \mathrm{Ag}$ particles, the scattering cross section is larger. Even if the absorption cross section were strictly zero, it would be possible for the $\mathrm{Ag}$ photo-oxidation reaction to occur in the coherent plasmon state. The quantum yield we quote is the ratio of the oxidation reaction rate to the scattering and absorption rates. The fundamental theoretical question of quantum yield in the presence of both absorption and scattering needs to be considered.

(44) Otto, A.; Futamata, M. Top. Appl. Phys. 2003, 85, 365

(45) Bastys, V.; Pastoriza-Santos, I.; Rodriguez-Gonzalez, B.; Vaisnoras, R.; Liz-Marzan, L. M. Adv. Funct. Mater. 2006, 16, 766.

(46) Van Hyning, D. L.; Zukoski, C. F. Langmuir 1998, 14, 7034.

(47) Redmond, P.; Hallock, A.; Brus, L. Nano Lett. 2005, 5, 131.

(48) Metraux, G. S.; Mirkin, C. A. Adv. Mater. 2005, 17, 412. 